\title{
Prediction of preterm labor by the level of serum magnesium using an optimized linear classifier
}

\author{
Soheila Aminimoghaddam*1미, Saeedeh Barzin Tond ${ }^{1}$, Alireza Mahmoudi Nahavandi ${ }^{2}$, Ahmadreza Mahmoudzadeh ${ }^{3}$, \\ Sepideh Barzin Tond ${ }^{4}$
}

Received: 24 Jul 2019

Published: 11 Apr 2020

\begin{abstract}
Background: This study investigates the possibility of predicting preterm labor by utilizing serum Magnesium level, BMI, and muscular cramp.

Methods: In this case-control study, 75 preterm and 75 term labor women are included. Different factors such as serum magnesium level, mother's age, infant's sex, mother's Body Mass Index (BMI), infant's weight, gravid, and muscular cramp experience are measured. Preterm labor is predicted by developing a linear discriminant model using Matlab, and the prediction accuracy is also computed.

Results: The results show that each of the studied variables has a significant correlation with preterm labor. The p-value between BMI and preterm labor is 0.005 , and by including the muscular cramp, it becomes less than 0.001 . The correlation between serum magnesium level and the preterm labor is less than 0.0001. Using these three significant variables, a linear discriminant function is developed, which improves the accuracy of predicting preterm labor.

Conclusion: The prediction error of preterm labor decreases from $31 \%$ (using only serum magnesium level) to $24 \%$ using the new proposed discriminant function. Based on this, it is suggested to use the optimized linear discriminant function to enhance the prediction of preterm labor, since the serum magnesium level cannot predict the preterm labor accurately.
\end{abstract}

Keywords: Premature labor, Prenatal diagnosis, Biomarkers, Optimized linear classifier, Magnesium level

\author{
Conflicts of Interest: None declared \\ Funding: None \\ *This work has been published under CC BY-NC-SA 1.0 license. \\ Copyright $\odot$ Iran University of Medical Sciences
}

Cite this article as: Aminimoghaddam S, Barzin Tond S, Mahmoudi Nahavandi A, Mahmoudzadeh A, Barzin Tond S. Prediction of preterm labor by the level of serum magnesium using an optimized linear classifier. Med J Islam Repub Iran. 2020 (11 Apr);34:32. https://doi.org/10.47176/mjiri.34.32

\section{Introduction}

Magnesium is an alkaline earth metal that its ion plays an important role in many enzymatic metabolic reactions (1). The magnesium- phosphate combination is the key feature of DNA and RNA. Magnesium is recorded in the form of serum magnesium level, which itself underestimates the right magnesium level about $70 \%$. Bones act as a buffer in regulating serum magnesium level and compensate for a decrease in extracellular magnesium level (2, 3).

Preterm labor accounts for 85 percent of infants' death,

Corresponding author:Dr Soheila Aminimoghaddam,aminimoghaddam.s@iums.ac.ir

1. School of Medicine, Iran University of Medical Sciences, Tehran, Iran

2. Department of Color Imaging and Color Image Processing, Institute for Color Science and Technology, Tehran, Iran

3. Zachry Department of Civil and Environmental Engineering, Texas A\&M University, College Station, TX, 77843-3136, USA

4. Department of Clinical Biochemistry, School of Medical Science, Shahid Beheshti University of Medical Sciences, Tehran, Iran which is comparable to child abnormality cases (4). Despite advances in perinatology and neonatology, premature infants are prone to suffer from diseases, such as blindness, lung dysfunction, problems in mental development and deafness (5).

Some studies have shown a significant correlation between preterm labor and magnesium level (6). However, there are other studies suggesting that there is not a significant improvement in taking magnesium (7). It is believed that the magnesium ions can compete with the Calcium

$\uparrow$ What is "already known” in this topic:

There are many causes for having preterm labor, including incompetent cervix, preterm rupture of membrane, diabetes of mother, and hypomagnesemia. A decrease of magnesium level may lead to preterm labor.

\section{$\rightarrow$ What this article adds:}

This study uses an optimized linear discriminant function to enhance the prediction of preterm labor. 
ions that are the main cause of uterine contractions.

The Genetic Algorithm as a modern technique of optimization has been used in many studies (8-12), including cardiovascular disease diagnosis (8), breast cancer diagnosis (9), Alzheimer's disease (10). Computer-aided diagnosis is under attention (CADx). This technique combines elements of computational intelligence and medical features as an aid for reducing diagnostic errors (13-15). Moreover, there are some studies reporting that preterm labor could not be predicted with sufficient accuracy from a single biochemical marker $(13,14)$. So it is wise to combine some biomarkers and factors to develop a new discriminant function for better prediction of preterm labor.

Although there have been many papers studying the effect of magnesium level on preterm labor, to the authors' knowledge, there has been no study concentrating on the effect of other factors such as mothers' age, BMI, Gravidae and child's gender on the preterm labor. These factors have been studied in the current paper as a means to better prenatal care. Moreover, using the Genetic Algorithm, a combined linear discriminant function is designed as an aid to better prediction of preterm labor.

\section{Methods}

This prospective cohort study was carried out on 75 women diagnosed as preterm labor between 28 and 37 weeks of gestation and 75 non-complicated pregnant at Akbar Abadi teaching hospital from October 2015 to December 2016.

Informed consent was obtained from all patients. The study protocol was approved by Iran University of Medical Sciences (IUMS) ethics committee with the identification number of IR.IUMS.REC1394.9211290002. The women in the case group were between 18 and 37 years old, with an average age of 27.5. The control group consisted of 75 non-complicated pregnant women with an average age of 28.5 (range: 19-43), which were admitted to the hospital for routine third-trimester visits.

Patients with multiple pregnancies, preeclampsia, gestational diabetes, preterm labor history, rupture of membrane, previous history of recurrent abortion, polyhydramnios, antepartum hemorrhage, congenital fetal malformation, premature rupture of membrane, cervical incompetence, uterine malformation, uterine myoma and magnesium supplements were excluded from the study.

The extracellular magnesium level was measured in the laboratory of Akbarabadi Hospital using Pars Azmon kit and autoanalyzer spectrophotometer. Venous blood samples $(2 \mathrm{cc})$ were taken from antecubital venous plexus and analyzed. Samples were poured into heparinized tubes and centrifuged for 10 minutes.

\section{Statistical analysis}

IBM SPSS 19 was used to conduct analysis and reporting the values. Independent samples t-test was used for comparing the variables, including age, BMI, magnesium level, and Gravidae. The sex of the neonates and the experience of muscular cramp in the preterm and term groups were evaluated with the Pearson $\chi 2$ test. The significance level of all statistical tests were adjusted to 0.05 .

In order to increase the performance of the diagnosis, a linear classifier was developed by minimizing the prediction error. The coefficients of the linear classifier were found using the Genetic Algorithm. The Migration, Crossover and Mutation parameters were set to $0.2,0.7$, and 0.1 , respectively. Fitness function was defined based on the sum of type 1 and type 2 classification errors (16). The generation was stopped when the mean and the best performance of the population reach the same amount. Fifty chromosomes were considered in each population. Each chromosome was composed of 4 genes representing coefficients for serum magnesium level, muscular cramp, BMI, and the bias of a linear classifier. Matlab R2013a software and its Optimization Toolbox were used for running the algorithm.

The Fitness Function is defined in

Equation 1. Small subscript numbers below each letter represent the dimension of the corresponding letter. In

Equation 1, "A" is the matrix of measured values of magnesium, the occurrence of cramp, BMI, and the bias. "c" represents the weights that are to be optimized, and " $\mathrm{f}$ " is the vector of values obtained for studying 150 women. The optimization stops when the average prediction error of the population reaches the minimum diagnosis error.

\section{Equation 1:}

$$
\begin{aligned}
\text { Fitness }=\arg \min _{c}\left(f_{150 * 1}\right. \\
\left.=A_{150 * 4} \cdot c_{4 * 1 \text { such that:diagnosis error } \rightarrow \text { min }}\right)
\end{aligned}
$$

Where $\mathrm{A}=\left[\mathrm{Mg}_{150^{*} 1}, \mathrm{Crump}_{150^{* 1},}, \mathrm{BMI}_{150^{* 1} 1}, \mathrm{I}_{150^{*} 1}\right]_{150 * 4}$

\section{Results}

Table 1 provides the statistics for the parameters. Sex types are reported in Male percentage. Gravidae 1 shows the percentage of cases admitted for the first-time labor within each of the case and control groups. Figure 1 illustrates the distribution of the Mother's age, BMI, and magnesium level within each group. The dark lines in the middle of the boxes are the median for each group. The deviation of each line from the center is due to the presence of extreme data points. It is obvious that the control group possesses a far outlier data.

Gravidae 1 is controlled for its effect on preterm labor. Pearson $\chi 2$ test was used for evaluating the uniformity of case and control group in term of Gravidae 1 and multiparous cases. Results showed that there is not a significant difference between case and control groups in terms of Gravidae $(\mathrm{p}=0.509)$.

Using an independent t-test, mother's age was compared between control and case groups as an indicator of the probability of preterm labor. Results showed that although the mother's age in preterm labor is lower than term labor, this difference is not significant $(\mathrm{p}=0.182)$. The independent sample t-test was conducted between the groups and showed that the mean of BMI in the control group was significantly higher than the case group $(p=0.005)$.

The prevalence of preterm labor in male and female in- 
Table 1. Statistics (Mean \pm Standard Error) of the studied parameters within case (Preterm) and control (Term) groups

\begin{tabular}{lcc}
\hline Variable & Preterm & Term \\
\hline Mother's Age & $27.51 \pm 0.51$ & $28.51 \pm 0.55$ \\
Gravidae 1 & $60.00 \%$ & $54.67 \%$ \\
Magnesium & $1.83 \pm 0.04$ & $2.15 \pm 0.05$ \\
BMI & $25.09 \pm 0.45$ & $26.81 \pm 0.41$ \\
Weight & $2097 \pm 63$ & $3295 \pm 35$ \\
Gestational Age & $33.27 \pm 0.28$ & $38.72 \pm 0.12$ \\
Muscular Cramp & $69.33 \%$ & $36.00 \%$ \\
Sex (Male \%) & $58.67 \%$ & $45.33 \%$ \\
\hline
\end{tabular}

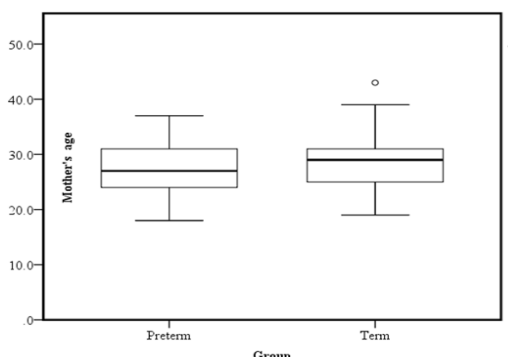

(a)

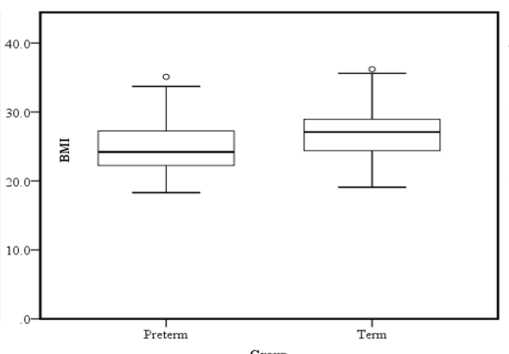

(b)

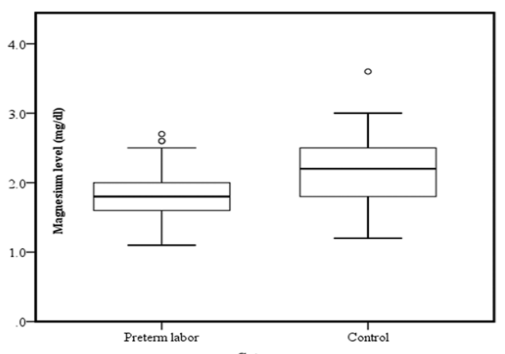

(c)

Fig. 1. Distribution of Mother's age (a) and BMI (b) and magnesium levels (c) of Control and Preterm labor

fants was studied using the Pearson $\chi 2$ test. Results failed to reject the assumption of having the same prevalence preterm labor among the two infant groups $(p=0.102)$. In other words, although there is more probability that male infants have premature birth on average (58.67\% in preterm labor group), it cannot be concluded that male infants are significantly more susceptible to the risk of premature birth.

The magnesium level in the case and control groups was compared using an independent samples t-test. Results showed that there is a significant difference between the magnesium level of the term and preterm groups $(p<0.001)$. It was revealed that the former group with the mean of $2.15 \mathrm{mg} / \mathrm{dl}$ has a significantly higher magnesium level than the later $(1.83 \mathrm{mg} / \mathrm{dl})$.

Using a Pearson $\chi 2$ test, muscular cramp was compared between the case and control groups. It was observed that there is a significantly higher rate of patients with muscle cramp history in the case group $(69.33 \%)$ compared to the control group $(36.00 \%)(\mathrm{p}<0.001)$. Analysis of BMI showed a significant difference between the case and control groups. It is worth noting that the BMI of the case group was $25.09 \pm 0.45$, and the BMI of the control group was $26.81 \pm 0.41$.

To study the association between the occurrence of hypomagnesemia with the occurrence of muscular cramp, the case group was divided into two subgroups. The first group had preterm labor cases with magnesium level lower than 1.9, and the second group had the cases with magnesium level greater than or equal to 1.9 . Pearson $\chi 2$ test showed that there is not any significant association between occurrences of hypomagnesemia and muscular cramp in the case group $(\mathrm{p}=0.525)$.

Different values of magnesium levels were scanned and tested to get the best criterion level. The results of the evaluation of prediction error have been shown in Fig. 2. It

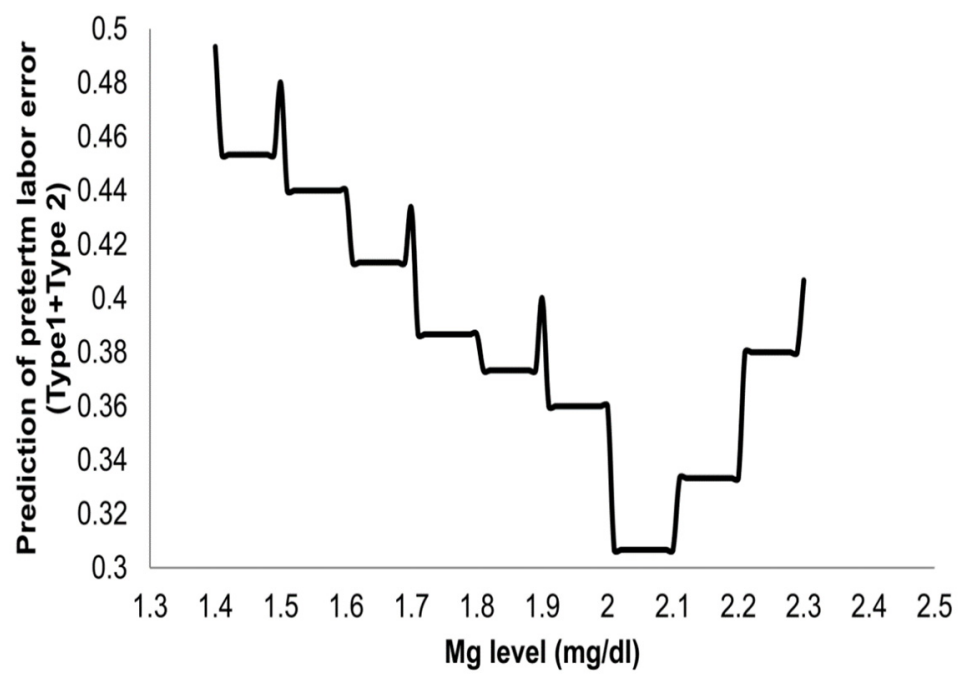

Fig. 2. Diagnosis error using different magnesium levels 

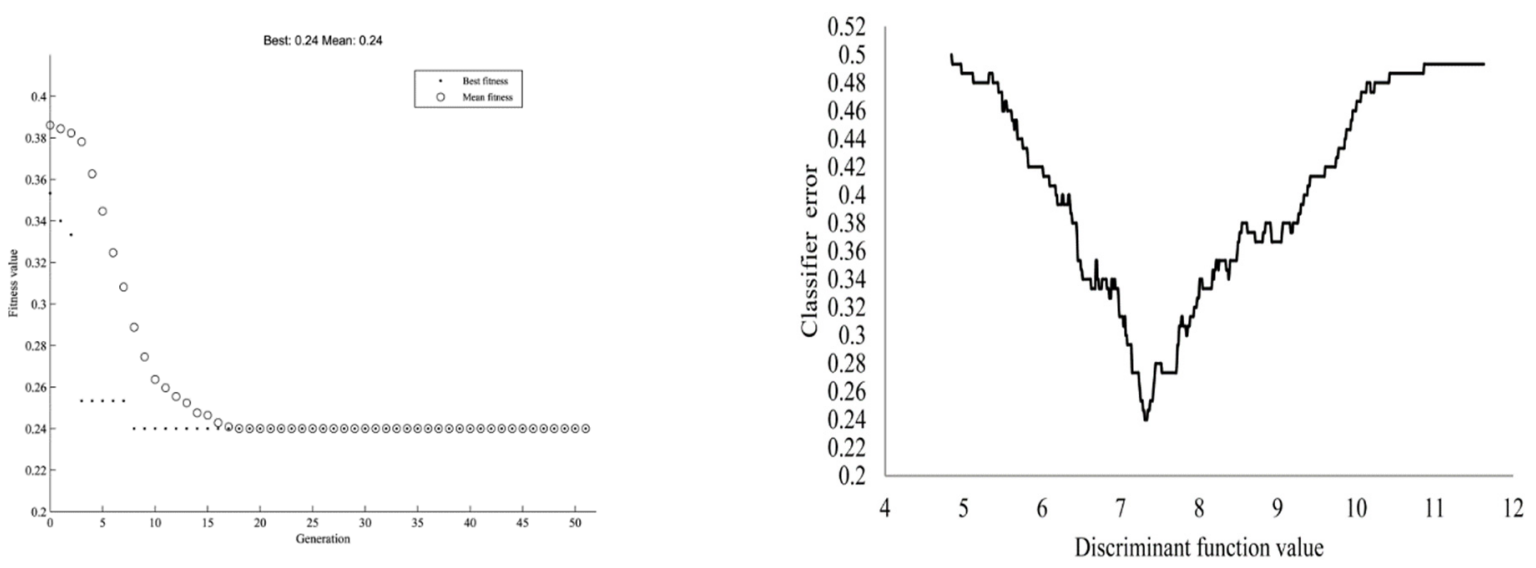

Fig. 3. (a) Stop criterion and (b) Classifier error vs. discriminant function value

shows that the minimum possible discrimination error occurs for the magnesium level ranging between 2 and 2.1 $\mathrm{mg} / \mathrm{dl}$. In other words, this tolerance limit can be used as a diagnostic criterion for preterm labor. Figure 2 shows that magnesium level could successfully predict diagnostic values for about $69.5 \%$ of the patients.

Prediction of preterm labor using the Genetic Algorithm leads to the optimized vector "c" as [1.7383 -0.7648 $0.12800 .8031]$. The optimized prediction error is $24 \%$ as shown in Figure 3 (a). The decision threshold is obtained at the minimum element of the vector "f" as shown in Equation 2 where $\mathrm{t}$ indicates transpose of a vector. From a different perspective, the minimum of the curve (discriminant function value $=7.3212$ ) can be used as a decision point for predicting the preterm labor.

Equation 2:

$$
f_{\min }=\min \left(A *\left[\begin{array}{llll}
1.7383 & -0.7648 & 0.1280 & 0.8031
\end{array}\right]^{t}\right)
$$

\section{Discussion}

The significant difference between serum magnesium level within the case and control groups of this study is comparable with the other studies in this regard $(6,17)$. The authors believe that the lower BMI of the case group is due to malnutrition of the preterm patients, as most of the patients were belong to lower social classes. This conclusion is in agreement with the results of Kosa et al. (18). However, they have reported that the probability of preterm labor increases if the case group patients enter from the "underweight" and "normal" to the "overweight" and "obese" categories (18).

Higher significant rates of muscular cramp, observed within the case group, are probably due to the lack of magnesium, which plays a buffering role in extracellular contractions (2). Meanwhile, the insignificant correlation between Hypomagnesemia (magnesium $<1.9$ ) and muscular cramp in the case group might be due to the wrong selection of magnesium cut-off point, which was also used by Shahid et al. (19). However, other studies proposed different magnesium levels, such as $1.16 \pm 0.22$ by Baloch et al. (20), 4.7 by Khani et al. (21), and $1.73 \pm 0.4$ by
Okunade et al. (22). Although many causes have been suggested for occurring preterm labor (23), serum magnesium level has been considered as one of the main reasons (2).

The selection of magnesium level, BMI, and muscular crump for prediction of preterm labor is due to their significant difference in term and preterm groups. It is a common technique in feature selection to take advantage of features that are significantly different between classes (24-26). The Genetic Algorithm can find the weights for the mentioned feature to enhance the prediction accuracy from $69.5 \%$ for magnesium level to $76 \%$. In summary, the results show that the magnesium level is a significant factor in diagnosing preterm labor. Also, using the developed discriminate function improves the accuracy of this detection.

\section{Conclusion}

This prospective cohort study investigates the effect of different parameters on preterm labor. Seventy-five women are diagnosed with preterm labor, along with 75 noncomplicated pregnant women are considered as the case and control groups, respectively. Different parameters are compared between the groups using statistical analysis methods, and a predictive model is developed using a Genetic Algorithm technique to improve the accuracy of predicting the occurrence of preterm labor. It is observed that the level of magnesium is significantly higher in the control group compared to the case group $(\mathrm{p}<0.001)$. Moreover, the Body Mass Index (BMI) is significantly lower in the case group $(p=0.005)$. A higher rate of muscular cramp is observed in the case group compared to the control group $(\mathrm{p}<0.001)$. Hypomagnesemia, as one of the most important causes of preterm labor, is also studied. The developed linear discriminant function confirmed that the error of preterm labor prediction could be decreased to $24 \%$.

\section{Funding}

The authors did not receive any funding for this project.

\section{Conflict of Interests}


The authors declare that they have no competing interests. References

1. Andreini C, Bertini I, Cavallaro G, Holliday GL, Thornton JM. Metal ions in biological catalysis: from enzyme databases to general principles. JBIC J Biol Inorg Chem. 2008;13(8):1205-18.

2. Dalton LM, Ní Fhloinn DM, Gaydadzhieva GT, Mazurkiewicz OM, Leeson H, Wright CP. Magnesium in pregnancy. Nutr Rev. 2016;74(9):549-57.

3. Arnaud MJ. Update on the assessment of magnesium status. Br J Nutr. 2008;99(S3):S24-36.

4. Goldenberg RL, Culhane JF, Iams JD, Romero R. Epidemiology and causes of preterm birth. Lancet. 2008;371(9606):75-84.

5. Chandiramani M, Tribe RM, Shennan AH. Preterm labour and prematurity. Obstet Gynaecol Reprod Med. 2007;17(8):232-7.

6. Uludağ EÜ, Gözükara IÖ, Kucur SK, Uluğ P, Özdeğirmenci Ö, Erkaya S. Maternal magnesium level effect on preterm labor treatment. J Matern Neonatal Med. 2014;27(14):1449-53.

7. Hsu WY, Wu CH, Hsieh CTC, Lo HC, Lin JS, Kao MD. Low body weight gain, low white blood cell count and high serum ferritin as markers of poor nutrition and increased risk for preterm delivery. Asia Pac J Clin Nutr. 2013;22(1):90-9.

8. Amma NGB. Cardiovascular disease prediction system using genetic algorithm and neural network. In: 2012 International Conference on Computing, Communication and Applications. IEEE; 2012. p. 1-5

9. Coroiu AM. Tuning model parameters through a genetic algorithm approach. In: 2016 IEEE 12th International Conference on Intelligent Computer Communication and Processing (ICCP). IEEE; 2016. p. 135-40.

10. Johnson P, Graham P, Wilson B, Macaulay L, Maruff P, Savage G, et al. Genetic algorithm with logistic regression for Alzheimer's disease diagnosis and prognosis. Alzheimer's Dement J Alzheimer's Assoc. 2013;9(4):P455-6.

11. Deekshatulu BL, Chandra P. Classification of heart disease using knearest neighbor and genetic algorithm. Procedia Technol. 2013;10:85-94.

12. Kaur N, Rattan M, Gill SS. Performance optimization of Broadwell$\mathrm{Y}$ shaped transistor using artificial neural network and Moth-flame optimization technique. Majlesi J Electr Eng. 2018;12(1):61-9.

13. Menon R, Torloni MR, Voltolini C, Torricelli M, Merialdi M, Betrán AP, et al. Biomarkers of spontaneous preterm birth: an overview of the literature in the last four decades. Reprod Sci. 2011;18(11):1046-70.

14. Kacerovsky M, Lenco J, Musilova I, Tambor V, Lamont R, Torloni $\mathrm{MR}$, et al. Proteomic biomarkers for spontaneous preterm birth: a systematic review of the literature. Reprod Sci. 2014;21(3):283-95.

15. Sahiner B, Chan H-P, Hadjiiski LM, Roubidoux MA, Paramagul C, Bailey JE, et al. Multi-modality CADx: ROC study of the effect on radiologists' accuracy in characterizing breast masses on mammograms and 3D ultrasound images. Acad Radiol. 2009;16(7):810-8.

16. Fukunaga K. Introduction to statistical pattern recognition. Elsevier; 2013.

17. Enaruna NO, Ande ABA, Okpere EE. Clinical significance of low serum magnesium in pregnant women attending the University of Benin Teaching Hospital. Niger J Clin Pract. 2013;16(4).

18. Kosa JL, Guendelman S, Pearl M, Graham S, Abrams B, Kharrazi $\mathrm{M}$. The association between pre-pregnancy BMI and preterm delivery in a diverse southern California population of working women. Matern Child Health J. 2011;15(6):772-81.

19. Shahid AR, Hosna AU, Tahmina HZ. Hypomagnesaemia in pregnancy: a predictor of preterm labour. J Dhaka Med Coll. 2010;19(1):51-7.

20. Baloch GH, Shaikh KH, Jaffary MH. Serum magnesium level during pregnancy. World Appl Sci J. 2012;17(8):1005-8.

21. Khani S, Shokrzadeh M, Karamoddini PK, Shahmohammadi S. The relationship between maternal serum magnesium level and preterm birth. Pakistan J Biol Sci. 2010;13(7):335.

22. Okunade KS, Oluwole AA, Adegbesan-Omilabu MA. A study on the association between low maternal serum magnesium level and preterm labour. Adv Med. 2014;2014.

23. Georgiou HM, Di Quinzio MKW, Permezel M, Brennecke SP. Predicting preterm labour: current status and future prospects. Dis Markers. 2015;2015.

24. Häfner M, Liedlgruber M, Uhl A, Vécsei A, Wrba F. Color treatment in endoscopic image classification using multi-scale local color vector patterns. Med Image Anal. 2012;16(1):75-86.

25. Aminimoghaddam S, Seifirad S, Abbasi Dezfouli G, Abbasi N, Zare Mehrjardi A, Razavi SM, et al. Case Report: Uterine Primitive Neuroectodermal Tumor. Arch Iran Med. 2015;18(4):260-262.

26. Ghaoomi M, Aminimoghaddam S, Safari H, Mahmoudzadeh A. Awareness and practice of cervical cancer and Pap smear testing in a teaching hospital in Tehran. Tehran Uni Med J TUMS Publ. 2016;74(3):183-9. 